\title{
Hemodynamic and analgesic effects of dexmedetomidine in patients undergoing major abdominal surgeries
}

\author{
Prof. Abdelrahman Hassan Abdelrahman, Prof. Zienab Diab, Dr. Raafat Ahmed \\ Salem, Islam Mokhtar Ahmed.
}

\begin{abstract}
Background: Dexmedetomidine, a highly selective $\alpha 2$-adrenoceptor agonist, is widely used in clinical anaesthesia, and intensive care unit. The aim of this study is to evaluate the hemodynamic and analgesic effects of dexmedetomidine in major abdominal surgeries.

Patients and methods: 40 patients for radical cystectomy in the age range of $40-65$ years participated in this prospective, randomized, double blinded, placebo controlled clinical study. Patients were randomly assigned to receive either Dexmeditomidine (Dex group) or normal saline (0.9\%) (Control group).patients hemodynamics, Recovery profile, and Pain intensity were evaluated.

Results Intraoperative mean arterial pressure and heart rate were significantly lower but stable in Dex group. Patients in Dex group had lower VAS scores.

Conclusion: Dexmedetomidine is effective in attenuating hemodynamic response to surgical stimulation. Patients in Dex group experienced less postoperative pain.
\end{abstract}

\section{Introduction}

Dexmedetomidine, a highly selective a2-adrenoceptor agonist, is widely used in clinical anaesthesia, intensive care unit (ICU) management and pain treatment as a sedative agent (Xianbao et al, 2013).

Surgical injury to tissue as in major abdominal surgeries causes a variety of profound physiologic reactions which are essential to the restoration of an organisms' homeostasis (Homburger JA et al, 2006).

Recent evidence suggests that DEX attenuates hemodynamic response to surgical stimulation (Tasdogan et al, 2009).

Intraoperative administration of dexmedetomidine during general anesthesia appeared to have a strong morphine-sparing effect in controlling postoperative acute pain (lee et al, 2016). intraoperative administration of dexmedetomidine seems to promote the analgesic property of morphine patient controlled analgesia, and patients consumed less morphine during the first 24hours after major abdominal surgery (Ge et al, 2015).

\begin{abstract}
Aim of the study:-
The aim of this study is to evaluate hemodynamic and analgesic effect of dexmedetomidine in major abdominal or pelvi abdominal surgeries and its impact on post operative recovery profile.
\end{abstract}

Patients and methods:

This prospective, randomized, double blinded, placebo controlled clinical study was approved by the Ethics and Research Committee of Sohag Faculty of Medicine, Sohag University. It was conducted at Sohag University Hospital between August 2015 and December 2016. Written informed consent was obtained from each patient preoperatively. The study included 40 patients in the age range of $40-65$ years.

The Inclusion criteria were as follows:-

Patients with an American Society of Anesthesiologists (ASA) physical status of I or II and scheduled for major abdominal or pelvi-abdominal surgery under general anesthesia were included in this study. 
SOHAG MEDICAL JOURNAL

Exclusion criteria included the followings:-

A history of drug or alcohol abuse, allergy to any of the study medications , 2nd or 3rd degree heart block, treatment with $\alpha 2$ agonists or antagonists, chronic use of antipsychotic medications, chronic use of anti-inflammatory drugs, labile hypertension, cardiac dysrhythmias, coronary artery disease, renal, hepatic or cognitive impairment.

Patients were randomly assigned to receive either Dexmeditomidine (Dex group) or normal saline $(0.9 \%)$ (Control group). Randomization and enrollment were done using sequentially numbered closed envelops. All patients assessment were performed by an anesthetist blinded to the drug used for the study and to reduce the selection and pretest biases another anesthetist prepared the study drug and wrapped the syringe pumps and tubing.

All patients were premeditated with atropine (0.02 mg IM) and midazolam $5 \mathrm{mg}$ IM. Upon arrival to operative room, base line measurements of mean arterial blood pressure (MAP), heart rate (HR), and oxygen saturation were obtained. Electrocardiographic (ECG) leads were applied for continuous ECG monitoring using a Life Scope Monitor (BSM-2353, Nihon Kohden - Japan). Two large bore intravenous cannulas were inserted in the right and left forearms.

After complete aseptic conditions central venous line was inserted in the right internal jugular or subclavian vein using Seldinger's technique for rapid infusion of fluids if needed. Radial artery on either side was cannulated after doing Allen's test (to make sure that there is adequate blood supply for the wrist and hand by the ulnar artery) for continuous blood pressure monitoring.
Hemodynamic and analgesic effects Islam Mokhtar Ahmed.et al

Patients were randomly divided into two groups:-

Patients in the dexmedetomidine group (Dex group) received a loading dose of dexmedetomidine at $1 \mu \mathrm{g} / \mathrm{kg}$ in $20 \mathrm{ml}$ of normal saline infused IV over 10 minutes followed by a maintenance infusion of $0.5 \mu \mathrm{g} / \mathrm{kg} / \mathrm{h}$ till the end of surgery, and patients in the control group received $20 \mathrm{ml}$ of normal saline infused IV over 10 minutes followed by a maintenance infusion of normal saline till the end of surgery.

Induction of general anesthesia in both groups was done by propofol (1.5-2 $\mathrm{mg} / \mathrm{kg})$ and fentanyl (2-5 $\mu \mathrm{g} / \mathrm{kg})$. Rocuronium (1 $\mathrm{mg} / \mathrm{kg}$ ) was used to facilitate endotracheal intubation. Patients were ventilated with an oxygen - air mixture $(\mathrm{FiO} 2=0.4)$ with EtCO2 stabilized at $30-35 \mathrm{mmHg}$.

Anesthesia was maintained by inhalational route with Sevoflourane in both groups. All patients in both groups received ondansetron $4 \mathrm{mg}$ prior to the end of surgery.

Patients were awakened and extubated in the operative room and were transferred to the PACU upon following simple commands. In the PACU, all patients received rescue morphine as indicated by visual analogue score $\geq 4$.

Data collection and measurements:Patient characteristics including age , sex, weight, ASA physical status, volume of blood loss and volume of blood transfused in addition to duration of anesthesia (time from the start of induction of anesthesia till discontinuation of inhalational anesthesia, reversal of muscle relaxant and tracheal extubation). And duration of surgery (time from the skin incision till the end of skin closure) were recorded.

Baseline measurements of heart rate, non - invasive mean arterial blood pressure, and $\mathrm{SpO} 2$ were obtained just prior to the start of the study drug 
SOHAG MEDICAL JOURNAL
Hemodynamic and analgesic effects Islam Mokhtar Ahmed.et al infusions, then heart rate and non invasive mean arterial blood pressure were recorded one minute before induction of anesthesia, one minutes after endotracheal intubation, intraoperatively every 15 minutes till the end of surgery, after complete recovery and postoperatively every 2 hours during the first 24 hours.

Recovery profile were assessed by measuring tracheal extubation time, time to eye opening, time to follow verbal commands, and time to have modified Alderte score $\geq 9$ was recorded.( White P et al, 1999).

Pain intensity was evaluated on $10 \mathrm{~cm}$ visual analogue scale (VAS) when 0 indicated no pain and 10 indicated sever pain.

The visual analogue scale (VAS) was recorded at 10, 30, 60, 90 minutes and at 6,12 , and 24 hours postoperatively.

Time to the first request of analgesia (VAS $>4)$ was recorded and the total dose of morphine that was given for each patient in the first 24 hours was recorded.

\section{Results}

A total of forty patients were enrolled in this prospective study; twenty patients received dexmedetomidine and twenty patients as control.

All patients underwent radical cystectomy with orthotopic diversion. Patients' demographic data, duration of anaesthesia and surgery, volume of blood loss, and volume of blood transfusion did not differ between the two groups (Table 1).

Table (1): Patients demographic data, duration of anesthesia and surgery, volume of blood loss, and volume of blood transfusion.

\begin{tabular}{|c|c|c|c|}
\hline & $\begin{array}{c}\text { Dex group } \\
(\mathrm{N}=20)\end{array}$ & $\begin{array}{c}\text { Control group } \\
(\mathrm{N}=20)\end{array}$ & *P-value \\
\hline Age (years) & $54.45 \pm 8.9$ & $54.65 \pm 8.5$ & 0.94 \\
\hline $\begin{array}{c}\text { Sex: } \\
\text { Male }\end{array}$ & $18(90)$ & $\begin{array}{c}17(85 \%) \\
3(15 \%)\end{array}$ & 0.4 \\
\hline $\begin{array}{c}\text { Female } \\
\text { Weight (kg) }\end{array}$ & $78 \pm 10.05$ & $79.5 \pm 7.76$ & 0.6 \\
\hline $\begin{array}{c}\text { Physical status } \\
\text { (ASA): } \\
\text { I }\end{array}$ & $8(40 \%)$ & $9(45 \%)$ & 0.23 \\
II & $12(60 \%)$ & $11(55 \%)$ & 0.45 \\
\hline $\begin{array}{c}\text { Duration of } \\
\text { anaesthesia } \\
\text { (hours) }\end{array}$ & $5.1 \pm 1.65$ & $5.28 \pm 1.45$ & 0.57 \\
\hline $\begin{array}{c}\text { Duration of } \\
\text { surgery (hours) }\end{array}$ & $4.71 \pm 1.03$ & $4.91 \pm 1.35$ & 0.36 \\
\hline $\begin{array}{c}\text { Volume of blood } \\
\text { loss (ml) }\end{array}$ & $1237.5 \pm 884.6$ & $1030 \pm 542$ & 0.91 \\
\hline $\begin{array}{c}\text { Volume of blood } \\
\text { transfusion (ml) }\end{array}$ & $940 \pm 857.4$ & $975.25 \pm 1070.6$ & \\
\hline
\end{tabular}

Data are presented as mean \pm standard deviation or number (\%).

Dex group $=$ dexmedetomidine group.

*P-value denotes statistical significance. 


\section{Intraoperative hemodynamic parameters:}

- Intraoperative heart rate (HR).

Intraoperative heart rate was significantly lower in Dex group at $30 \mathrm{~min}, 45 \mathrm{~min}, 60$ $\mathrm{min}, 75 \mathrm{~min}, 90 \mathrm{~min}, 105 \mathrm{~min}$ and $120 \mathrm{~min}$ as shown in table(2) and figure (1).

Table (2): Intraoperative heart rate (HR).

\begin{tabular}{|c|c|c|c|}
\hline & $\begin{array}{c}\text { Dex group } \\
\mathrm{N}=20\end{array}$ & $\begin{array}{c}\text { Control group } \\
\mathrm{N}=20\end{array}$ & P-value \\
\hline HR at baseline & $77.9 \pm 10.93$ & $78.9 \pm 7.8$ & 0.741 \\
\hline $\begin{array}{c}\text { HR at one minute } \\
\text { after induction }\end{array}$ & $77.85 \pm 10.48$ & $77.4 \pm 8.2$ & 0.881 \\
\hline $\begin{array}{c}\text { HR at one minute } \\
\text { after intubation }\end{array}$ & $77.1 \pm 12.4$ & $76.7 \pm 8$ & 0.893 \\
\hline HR at 15 min & $77.1 \pm 14.6$ & $77.2 \pm 8.3$ & 0.979 \\
\hline HR at 30 min & $69.4 \pm 7.3$ & $76.4 \pm 6.5$ & $0.003^{*}$ \\
\hline HR at 45 min & $68.3 \pm 7.7$ & $76.8 \pm 7.6$ & $0.001^{*}$ \\
\hline HR at 60 min & $69.1 \pm 7.6$ & $75.7 \pm 6.4$ & $0.005^{*}$ \\
\hline HR at 75 min & $66.6 \pm 7.4$ & $75.1 \pm 6.3$ & $0.001^{*}$ \\
\hline HR at 90 min & $65.8 \pm 7.9$ & $74.5 \pm 7.1$ & $0.001^{*}$ \\
\hline HR at 105 min & $66.3 \pm 8.9$ & $75.9 \pm 8.1$ & $0.001^{*}$ \\
\hline HR at 120 min & $65.7 \pm 10.5$ & $76.4 \pm 9.1$ & $0.001^{*}$ \\
\hline HR at 135 min & $72.9 \pm 12$ & $76.4 \pm 11$ & 0.350 \\
\hline HR at 150 min & $75 \pm 11.9$ & $77.2 \pm 9$ & 0.523 \\
\hline HR at 165 min & $75.7 \pm 9.5$ & $80.7 \pm 7.3$ & 0.069 \\
\hline HR at 180 min & $78 \pm 10.7$ & $82.4 \pm 7.1$ & 0.118 \\
\hline HR at 195 min & $81.4 \pm 9.1$ & $85.8 \pm 6.7$ & 0.181 \\
\hline HR at 210 min & $73.9 \pm 12$ & $78.4 \pm 11$ & 0.350 \\
\hline HR at 225 min & $76 \pm 11.9$ & $77.2 \pm 9$ & 0.523 \\
\hline HR at 240 min & $76.7 \pm 9.5$ & $81.7 \pm 7.3$ & 0.069 \\
\hline HR at recovery & $72.9(12)$ & $76.4(11)$ & 0.35 \\
\hline
\end{tabular}

Data are presented as mean \pm standard deviation.

Dex group $=$ dexmedetomidine group.

*P-value $<0.05$ denotes statistical significance versus the control group.

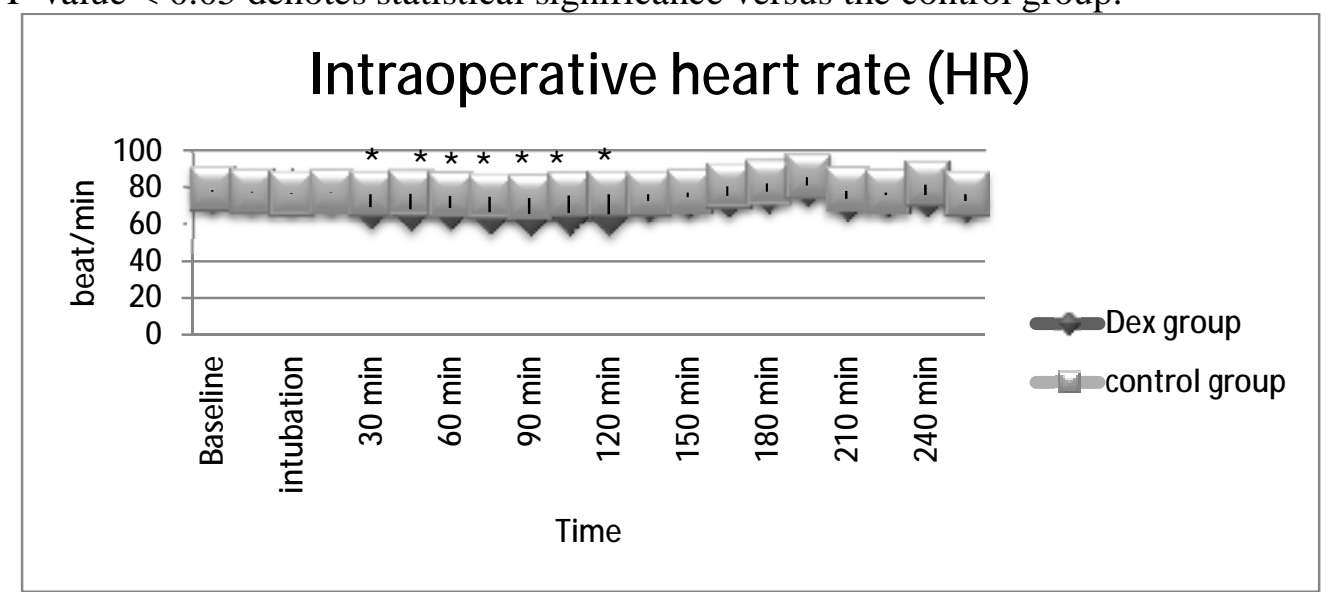

Figure (1): Intraoperative heart rate (HR).

Data are presented as mean.

Dex group $=$ dexmedetomidine group.

*P-value $<0.05$ denotes statistical significance versus the control group.

- Intraoperative mean arterial pressure MAP. 
SOHAG MEDICAL JOURNAL
Hemodynamic and analgesic effects Islam Mokhtar Ahmed.et al

Intraoperative mean arterial pressure was significantly lower in Dex group after induction, at $15 \mathrm{~min}, 45 \mathrm{~min}, 60 \mathrm{~min}$ and $120 \mathrm{~min}$ as shown in table (3) and figure(2)

Table (3): Intraoperative mean arterial pressure MAP.

\begin{tabular}{|c|c|c|c|}
\hline & $\begin{array}{l}\text { Dex group } \\
\mathrm{N}=20\end{array}$ & $\begin{array}{l}\text { Control group } \\
\quad \mathrm{N}=20\end{array}$ & P-value \\
\hline MAP at baseline & $111(9.2)$ & $110.3(8.2)$ & 0.766 \\
\hline $\begin{array}{l}\text { MAP at } \\
\text { induction }\end{array}$ & $100.1(6.5)$ & $104(5.3)$ & $0.045^{*}$ \\
\hline $\begin{array}{l}\text { MAP at } \\
\text { intubation }\end{array}$ & $92.2(5.6)$ & $92.9(11.2)$ & 0.818 \\
\hline MAP at $15 \mathrm{~min}$ & $89.7(8.3)$ & $101(5.2)$ & $<0.001 *$ \\
\hline MAP at $30 \mathrm{~min}$ & $94.1(6.2)$ & $93.5(3.6)$ & 0.734 \\
\hline MAP at $45 \mathrm{~min}$ & $92.8(9.7)$ & $104.8(6.1)$ & $<0.001 *$ \\
\hline MAP at $60 \mathrm{~min}$ & $89.2(4.1)$ & $94(3.1)$ & $<0.001 *$ \\
\hline MAP at 75 min & $82.6(5.4)$ & $82(3.9)$ & 0.666 \\
\hline MAP at $90 \mathrm{~min}$ & $80.2(7.4)$ & $79.2(5.8)$ & 0.638 \\
\hline MAP at $105 \mathrm{~min}$ & $81.5(7.2)$ & $82.6(5.8)$ & 0.599 \\
\hline MAP at $120 \mathrm{~min}$ & $78.1(7.8)$ & $87.6(4.3)$ & $<0.001 *$ \\
\hline MAP at $135 \mathrm{~min}$ & $88.1(10.8)$ & $91.7(9.5)$ & 0.277 \\
\hline MAP at $150 \mathrm{~min}$ & $94.9(9.2)$ & $91.2(9.3)$ & 0.207 \\
\hline MAP at $165 \mathrm{~min}$ & $98.2(6.5)$ & $97.5(10.1)$ & 0.803 \\
\hline MAP at $180 \mathrm{~min}$ & $97.7(7.9)$ & $100.5(9.1)$ & 0.299 \\
\hline MAP at $195 \mathrm{~min}$ & $100.2(7.9)$ & $101.1(10.6)$ & 0.520 \\
\hline MAP at $210 \mathrm{~min}$ & $95.9(9.2)$ & $90.2(9.3)$ & 0.207 \\
\hline MAP at $225 \mathrm{~min}$ & $97.2(6.5)$ & $95.5(10.1)$ & 0.803 \\
\hline MAP at $240 \mathrm{~min}$ & $96.7(7.9)$ & $101.5(9.1)$ & 0.299 \\
\hline MAP at recovery & $100.2(7.9)$ & $102.1(10.6)$ & 0.520 \\
\hline
\end{tabular}

Data are presented as mean \pm standard deviation.

Dex group $=$ dexmedetomidine group.

$* \mathrm{P}$-value $<0.05$ denotes statistical significance versus the control group.

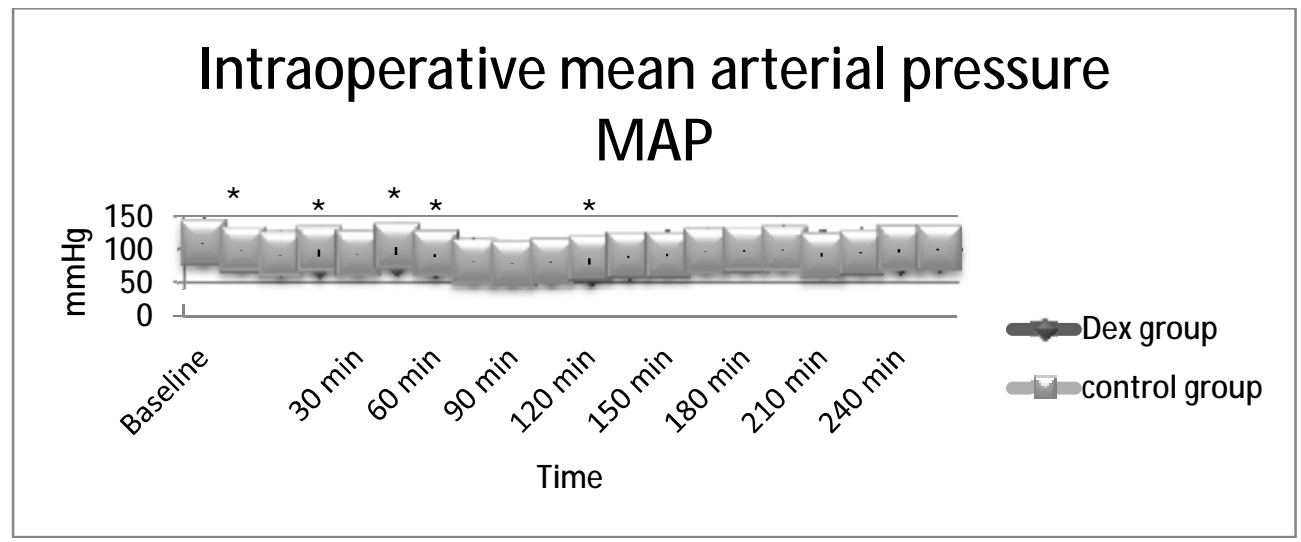

Figure (2): Intraoperative mean arterial pressure MAP.

Data are presented as mean.

Dex group $=$ dexmedetomidine group.

*P-value $<0.05$ denotes statistical significance versus the control group.

Postoperative hemodynamic parameters:

Postoperative heart rate (HR). There was no significant difference between either groups in postoperative heart rate. 
SOHAG MEDICAL JOURNAL
Hemodynamic and analgesic effects Islam Mokhtar Ahmed.et al

Table (4): Postoperative heart rate (HR).

\begin{tabular}{|c|c|c|c|}
\hline & $\begin{array}{c}\text { Dex group } \\
\mathrm{N}=20\end{array}$ & $\begin{array}{c}\text { Control group } \\
\mathrm{N}=20\end{array}$ & P-value \\
\hline HR at $2 \mathrm{hr}$ & $73 \pm 9.9$ & $75.2 \pm 7$ & 0.51 \\
\hline HR at $4 \mathrm{hr}$ & $74.3 \pm 10.9$ & $76.2 \pm 8.1$ & 0.49 \\
\hline HR at $6 \mathrm{hr}$ & $75.1 \pm 11.9$ & $77.1 \pm 9.3$ & 0.52 \\
\hline HR at $8 \mathrm{hr}$ & $75.5 \pm 7.9$ & $78.2 \pm 6.4$ & 0.52 \\
\hline HR at $10 \mathrm{hr}$ & $75.7 \pm 9.5$ & $80.7 \pm 7.3$ & 0.06 \\
\hline HR at $12 \mathrm{hr}$ & $76.6 \pm 8.1$ & $82.7 \pm 6.3$ & 0.09 \\
\hline HR at $14 \mathrm{hr}$ & $75.9 \pm 7.2$ & $81.3 \pm 5.4$ & 0.1 \\
\hline HR at $16 \mathrm{hr}$ & $78 \pm 10.7$ & $80.4 \pm 7.1$ & 0.12 \\
\hline HR at $18 \mathrm{hr}$ & $77.6 \pm 6.3$ & $81.2 \pm 6.1$ & 0.18 \\
\hline HR at $20 \mathrm{hr}$ & $79.2 \pm 10.7$ & $80.3 \pm 7.2$ & 0.17 \\
\hline HR at $22 \mathrm{hr}$ & $82.4 \pm 9.1$ & $83.8 \pm 6.7$ & 0.15 \\
\hline HR at $24 \mathrm{hr}$ & $83.5 \pm 6.1$ & $80.8 \pm 7.7$ & 0.14 \\
\hline
\end{tabular}

Data are presented as mean \pm standard deviation.

Dex group = dexmedetomidine group.

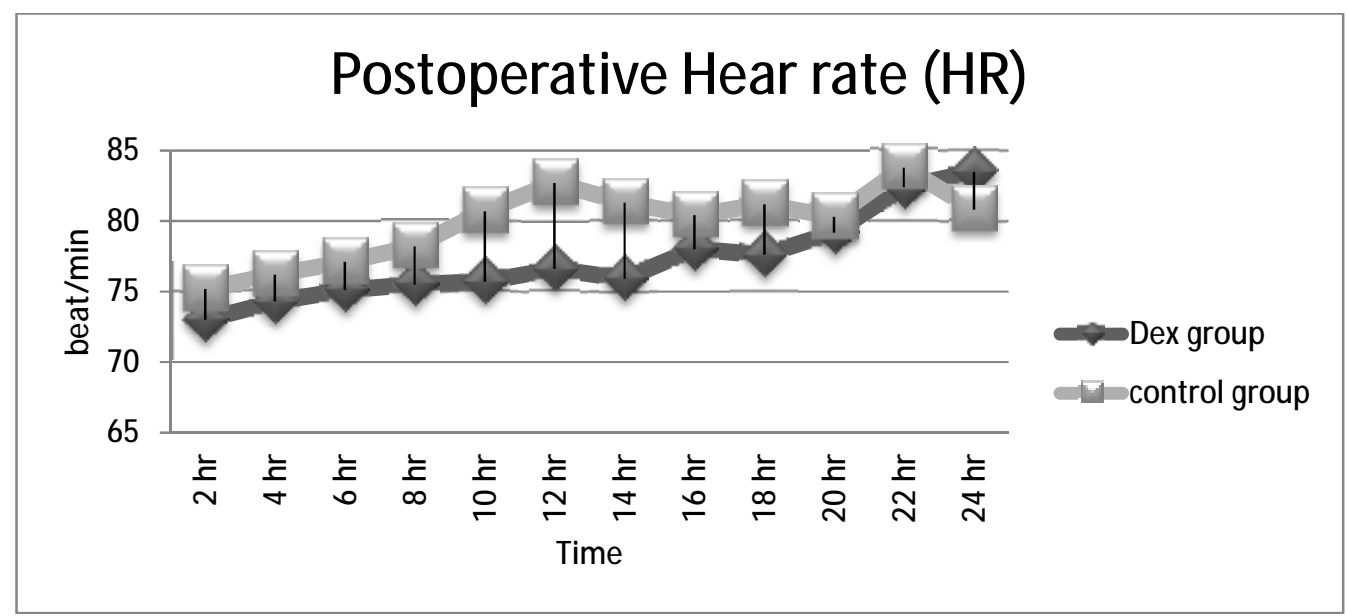

Figure (3): Postoperative heart rate (HR).

Data are presented as mean.

Dex group $=$ dexmedetomidine group.

Postoperative mean arterial pressure (MAP). There was no significant difference between either groups in Postoperative mean arterial pressure MAP. 
SOHAG MEDICAL JOURNAL
Hemodynamic and analgesic effects Islam Mokhtar Ahmed.et al

Table (5): Postoperative mean arterial pressure (MAP).

\begin{tabular}{|c|c|c|c|}
\hline & $\begin{array}{c}\text { Dex group } \\
\mathrm{N}=20\end{array}$ & $\begin{array}{c}\text { Control group } \\
\mathrm{N}=20\end{array}$ & P-value \\
\hline MAP at $2 \mathrm{hr}$ & $94.9 \pm 9.2$ & $91.2 \pm 9.3$ & 0.207 \\
\hline MAP at $4 \mathrm{hr}$ & $94.2 \pm 6.5$ & $92.1 \pm 10.1$ & 0.803 \\
\hline MAP at $6 \mathrm{hr}$ & $95.7 \pm 7.9$ & $93.5 \pm 9.1$ & 0.299 \\
\hline MAP at $8 \mathrm{hr}$ & $92.2 \pm 7.9$ & $94.1 \pm 10.6$ & 0.520 \\
\hline MAP at $10 \mathrm{hr}$ & $98.2 \pm 6.5$ & $97.5 \pm 10.1$ & 0.803 \\
\hline MAP at $12 \mathrm{hr}$ & $96.6 \pm 8.1$ & $96.7 \pm 6.3$ & 0.09 \\
\hline MAP at $14 \mathrm{hr}$ & $94.9 \pm 7.2$ & $95.3 \pm 5.4$ & 0.1 \\
\hline MAP at $16 \mathrm{hr}$ & $93.7 \pm 7.9$ & $94.5 \pm 9.1$ & 0.299 \\
\hline MAP at $18 \mathrm{hr}$ & $92.6 \pm 6.3$ & $93.2 \pm 6.1$ & 0.18 \\
\hline MAP at $20 \mathrm{hr}$ & $92 \pm 10.7$ & $94.3 \pm 7.2$ & 0.17 \\
\hline MAP at $22 \mathrm{hr}$ & $100.2 \pm 7.9$ & $102.1 \pm 10.6$ & 0.520 \\
\hline MAP at $24 \mathrm{hr}$ & $101.5 \pm 6.1$ & $102.8 \pm 7.7$ & 0.14 \\
\hline
\end{tabular}

Data are presented as mean \pm standard deviation.

Dex group $=$ dexmedetomidine group.

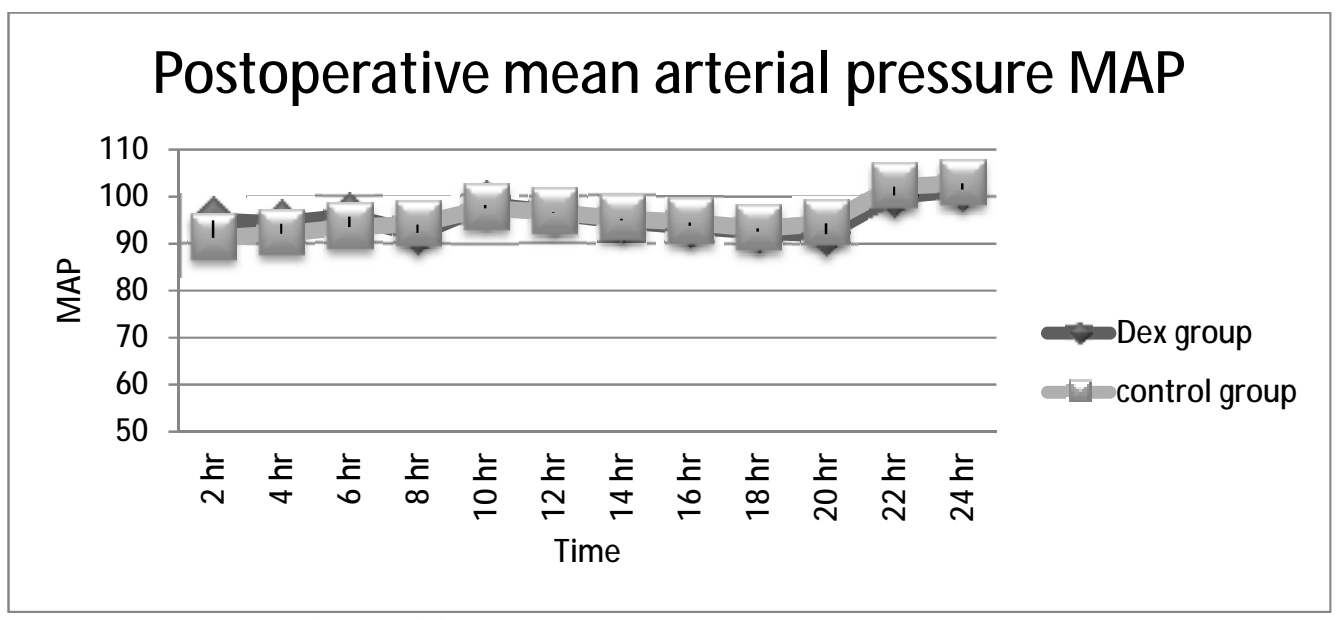

Figure (4): Postoperative mean arterial pressure MAP.

Data are presented as mean. Dex group $=$ dexmedetomidine group.

\section{Recovery profile:}

Recovery profile was evaluated by measuring endotracheal extubation time, time to eye opening, time to following verbal command, and time to have modified Alderet score $\geq 9$ which were longer in Dex group than in control group but without statistically significant difference between both groups as shown in table (7). 
SOHAG MEDICAL JOURNAL
Hemodynamic and analgesic effects Islam Mokhtar Ahmed.et al

Table (6): Recovery profile.

\begin{tabular}{|l|c|c|c|}
\hline & $\begin{array}{c}\text { Dex group } \\
\mathrm{N}=20\end{array}$ & $\begin{array}{c}\text { Control group } \\
\mathrm{N}=20\end{array}$ & P-value \\
\hline $\begin{array}{l}\text { Extubation time } \\
\text { (min.) }\end{array}$ & $20.3 \pm 14.79$ & $19.6 \pm 10.63$ & 0.86 \\
\hline $\begin{array}{l}\text { Time to eye opening } \\
\text { (min.) }\end{array}$ & $22.9 \pm 5.96$ & $22.2 \pm 6.2$ & 0.43 \\
\hline $\begin{array}{l}\text { Time to follow } \\
\text { verbal commands } \\
\text { (min.) }\end{array}$ & $28.2 \pm 12.72$ & $28.65 \pm 11.61$ & 0.91 \\
\hline $\begin{array}{l}\text { Time to have } \\
\text { modified Alderete } \\
\text { score } \geq 9 \text { (min) }\end{array}$ & $30.6 \pm 16.92$ & $26.95 \pm 11.72$ & 0.72 \\
\hline
\end{tabular}

Data are presented as mean \pm standard deviation.

Dex group = dexmedetomidine group.

Postoperative visual analogue scale VAS (table 7, figure 5):

Patients receiving dexmedetomidine had significant less VAS score at 60, 90, 120 minutes, and 6 hours postoperatively as shown in table (8) and figure (6).

Table (7): Postoperative VAS

\begin{tabular}{|c|c|c|c|}
\hline & $\begin{array}{c}\text { Dex group } \\
\mathrm{N}=20\end{array}$ & $\begin{array}{c}\text { Control group } \\
\mathrm{N}=20\end{array}$ & P-value \\
\hline VAS at $10 \mathrm{~min}$ & $3.2 \pm 0.7$ & $4.1 \pm 1.5$ & 0.351 \\
\hline VAS at $30 \mathrm{~min}$ & $4 \pm 1.2$ & $4.1 \pm 1.3$ & 0.700 \\
\hline VAS at $60 \mathrm{~min}$ & $4.1 \pm 1.7$ & $5.5 \pm 1$ & $0.003^{*}$ \\
\hline VAS at $90 \mathrm{~min}$ & $4.1 \pm 0.8$ & $5.6 \pm 1.5$ & $<0.001^{*}$ \\
\hline VAS at $120 \mathrm{~min}$ & $4.3 \pm 1$ & $5.3 \pm 1.6$ & $0.02^{*}$ \\
\hline VAS at $6 \mathrm{~h}$ & $4.7 \pm 0.5$ & $5.5 \pm 1.2$ & $0.008^{*}$ \\
\hline VAS at $12 \mathrm{~h}$ & $4.4 \pm 1.3$ & $5.2 \pm 2$ & 0.134 \\
\hline VAS at $24 \mathrm{~h}$ & $4.7 \pm 0.5$ & $4.9 \pm 1.9$ & 0.657 \\
\hline
\end{tabular}

Data are presented as mean \pm standard deviation.

Dex group $=$ dexmedetomidine group.

*P-value $<0.05$ denotes statistical significance versus the control group.

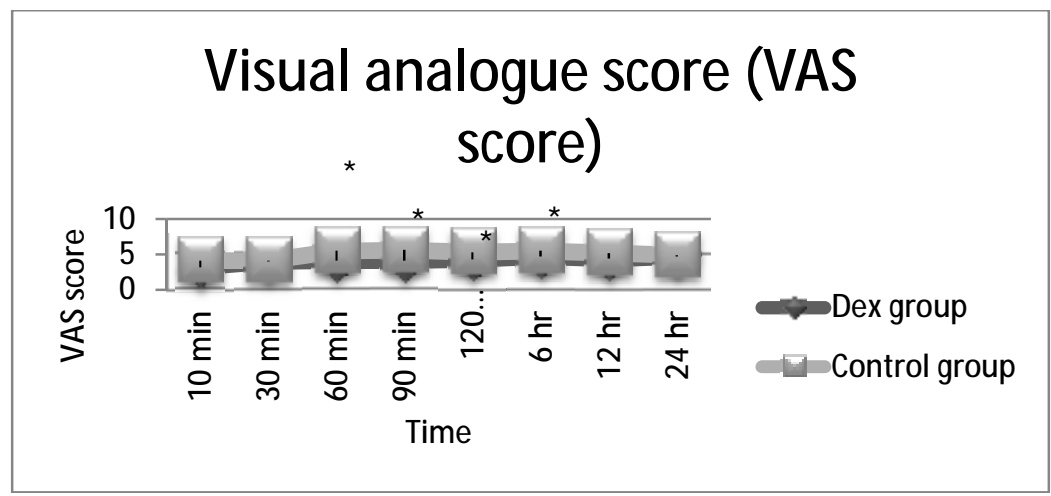

Figure (5): Postoperative visual analogue score (VAS score).

Data are presented as mean.

Dex group $=$ dexmedetomidine group.

*P-value $<0.05$ denotes statistical significance versus the control group.

Time to first analgesic requirment was prolonged in Dex group than the control group (P-value <0.001). Patients in Dex group required less total dose of morphine in postoperative period than control group (P-value $<0.001)$ as shown in table $(8)$. 
Table (8): Time to first analgesic requirement and total dose of morphine.

\begin{tabular}{|l|c|c|c|}
\hline & $\begin{array}{c}\text { Dex group } \\
\mathrm{N}=20\end{array}$ & $\begin{array}{c}\text { Control group } \\
\mathrm{N}=20\end{array}$ & P-value \\
\hline $\begin{array}{l}\text { Time to first } \\
\text { analgesic } \\
\text { requirement }\end{array}$ & $30 \pm 2.1$ & $8 \pm 2.5$ & $<0.001^{*}$ \\
\hline $\begin{array}{l}\text { postoperative total } \\
\text { dose of morphine }\end{array}$ & $20 \pm 3.2$ & $40 \pm 7.4$ & $<0.001^{*}$ \\
\hline
\end{tabular}

Data are presented as mean \pm standard deviation.

Dex group $=$ dexmedetomidine group.

$*$ P-value $<0.05$ denotes statistical significance versus the control group.

\section{Discussion}

Dexmedetomidine is a centrally acting $\alpha 2$-adrenergic agonist reducing norepinephrine turnover and decreasing central sympathetic outflow (kang et al, 2013).

The stress response to surgery is a neuroendocrine response to surgical trauma. It is characterized by increases in catecholamine with hemodynamic consequences (O'Riain et al., 2005).

Abdominal surgeries are associated with major alterations in haemodynamic response and marked post-operative pain (Herroeder et al, 2007).

In the present study, the basal readings of HR and MAP were within normal physiological ranges and with no significant difference between both groups. Patients received dexmedetomidine had significant lower MAP at induction but with haemodynamic stability. Jaakola et al. showed that dexmedetomidine attenuated the increase in $\mathrm{HR}$ and MAP during intubation (Jaakola et al., 1992).

HR and MAP were significantly lower in intraoperative period in Patients received dexmedetomidine. These results were in agreement with that reported by (Yacout et al., 2012). No patient in dexmedetomidine group required intervention for haemodynamic instability. In postoperative period, we found no significant difference between both groups in HR and MAP.
Dexmedetomidine group had lower mean heart rate and mean blood pressure compared to Placebo group after endoscopic sinus surgery (Tang et al, 2015). In comparison between dexmedetomidine and placebo, postoperative changes in mean HR between two groups were a statistically significant in cases of laparoscopic cholecystectomy (Manne et al, 2014).

In the cardiac surgery patients, no significant hemodynamics difference throughout recovery periods or undesirable hemodynamic effects (Hall et al, 2000).

We found no significant difference between both groups in extubation time and recovery time. Previous studies suggested that dexmedetomidine was associated with significantly longer times to extubation and recovery from anaesthesia (Ozcan et al., 2012).

Abel Rahman et al. found no significant difference of extubation and recovery between dexmedetomidine and placebo (Abel Rahman et al., 2014). In contrast, Nasreen et al. reported reduced recovery time in dexmedetomidine compared with placebo (Nasreen et al., 2009).

The efficacy of dexmedetomidine in alleviating postoperative pain was focused on the immediate postoperative period. This may be related to the pharmacokinetics of dexmedetomidine (Hwang et al, 2015). Arain and Ebert reported 
significant less analgesics need in postoperative period in patients received dexmedetomidine during surgery (Arain and Ebert, 2002). Yacout et al. reported lower VAS and less consumption of analgesics in Patients received dexmedetomidine compared to placebo. In the present study, VAS for pain score was significantly less in patients received dexmedetomidine. Analgesic effect of dexmedetomidine may be explained by activation of $\alpha 2$-adrenergic receptors in the dorsal horn of the spinal cord, which inhibits the release of substance $\mathrm{P}$, a nociceptive mediator, resulting in potentiation of opioid induced analgesia (Nelson et al., 2003).

Opioids especially morphine are widely used for postoperative pain control. To combat the side effects, such as nausea, vomiting, and itching, studies have sought to identify novel drugs or to generate more information regarding combining the currently available drugs to reduce morphine consumption(Movafegh et al, 2012, George et al, 2014).

In this study, patients in Dex group required significantly less total dose of morphine in postoperative period than control group. Previous studies declared the efficacy of intraoperative dexmedetomidine to reduce postoperative morphine consumption (Fan et al, 2017, Jessen et al, 2016). In previous study, patients received dexmedetomidine had an increased time to first request of postoperative morphine patients after abdominal surgery under general anaesthesia (Fan et al, 2017). In another study, the first postoperative request for sufentanil analgesia was delayed in patients given intranasal dexmedetomidine( Wu et al, 2016). Patients receiving dexmedetomine had longer time to first morphine request after radical mastectomy (Fan et al, 2017).

\section{Conclusion:}

Dexmedetomidine is effective in attenuating hemodynamic response to surgical stimulation. Patients in Dex group experienced less postoperative pain.

\section{References}

1. Abel Rahman, N. I., Fouad E. A., Ahmed, A., Youness, A. R. Efficacy of different dexmedetomidine regimens in producing controlled hypotensive anesthesia during functional endoscopic sinus surgery. Egyptian Journal of Anaesthesia 2014, 30, 339345 .

2. Arain S. R. and Ebert, T. J. The efficacy, side effects, and recovery characteristics of dexmedetomidine versus propofol when used for intraoperative sedation. Anesth Analg 2002, 95, 461-6, table of contents.

3. Fan, W, Yang, H, Sun, Y, Zhang, J, Li, G, Zheng, Y, and Liu, Y, Comparison of the pro-postoperative analgesia of intraoperative dexmedetomidine with and without loading dose following general anesthesia: A prospective, randomized, controlled clinical trial. Medicine (Baltimore), 2017. 96(7): p. e6106.

4. Ge, DJ, Qi, B, Tang, G, and Li, JY, Intraoperative Dexmedetomidine Promotes Postoperative Analgesia and Recovery in Patients after Abdominal Colectomy: A CONSORT-Prospective, Randomized, Controlled Clinical Trial. Medicine (Baltimore), 2015. 94(43): p. e1727.

5. George, RB, McKeen, DM, Andreou, $\mathrm{P}$, and Habib, AS, A randomized placebo-controlled trial of two doses of pregabalin for postoperative analgesia in patients undergoing abdominal hysterectomy. Can J Anaesth, 2014. 61(6): p. 551-7.

6. Herroeder, S., Pecher, S., Schonherr, M., Kaulitz. Systemic lidocaine shortens length of hospital stay after colorectal surgery: a double-blinded, 
SOHAG MEDICAL JOURNAL

Vol. 21 No.2 July 2017

randomized, placebo-controlled trial. Ann Surg 2007, 246, 192-200.

7. Homburger JA, Meiler SE. Anesthesia drugs, immunity, and long- erm outcome. Curr OpinAnaesthesiol. 2006; 19:423-8.

8. Hwang W, Lee J, Park J, Joo J. Dexmedetomidine versus remifentanil in postoperative pain control after spinal surgery: a randomized controlled study. BMC Anesthesiol. 2015 Feb 24; 15:21.

9. Jaakola, M. L., Kanto, J., Kallio, A., Scheinin. Dexmedetomidine reduces intraocular pressure, intubation responses and anaesthetic requirements in patients undergoing ophthalmic surgery. Br J Anaesth 1992, 68, 570-5.

10. Jessen Lundorf, L, Korvenius Nedergaard, H, and Moller, AM, Perioperative dexmedetomidine for acute pain after abdominal surgery in adults. Cochrane Database Syst Rev, 2016. 2: p. Cd010358.

11. Kang, S. H. et al. Effects of dexmedetomidine on inflammatory responses in patients undergoing laparoscopic cholecystectomy. Acta Anaesthesiol Scand. 57, 480-7 (2013).

12. Lee, J, Park, J, JOO, J. Dexmedetomidine versus remifentanil in postoperative pain control after spinal surgery: a randomized controlled study. BMC Anesthesiol 2015, 15, 21.

13. Manne, GR, Upadhyay, MR, and Swadia, V, Effects of low dose dexmedetomidine infusion on haemodynamic stress response, sedation and post-operative analgesia requirement in patients undergoing laparoscopic cholecystectomy. Indian J Anaesth, 2014. 58(6): p. 726-31.

14. Movafegh, A, Shoeibi, G, Ansari, M, Sadeghi, M, Azimaraghi, O, and Aghajani, Y, Naloxone infusion and post-hysterectomy morphine consumption: a double-blind, placebo-
Hemodynamic and analgesic effects

Islam Mokhtar Ahmed.et al

controlled study. Acta Anaesthesiol Scand, 2012. 56(10): p. 1241-9.

15. Nelson, B. A. Laura E., Lu, M., Guo, M., Saper, D. The a2-Adrenoceptor Agonist Dexmedetomidine Converges on an Endogenous Sleep-promoting Pathway to Exert Its Sedative Effects. Anesthesiology 2003, 98, 428-436.

16. O'Riain, S, Buggy, D, Kerin, M, Watson, R. Inhibition of the stress response to breast cancer surgery by regional anesthesia and analgesia does not affect vascular endothelial growth factor and prostaglandin E2. Anesth Analg 2005, 100, 244-9.

17. Ozcan, A., Ozcan, N., Yalcin, F. \& Bascar, H. Comparison of the effects of fentanyl, remifentanil, and dexmedetomidine on neuromuscular blockade. J Anesth 2012, 26, 196-9.

18. Tang, C, Huang, $X$, Kang, F, Chai, $X$, Wang, S, Yin, G, Wang, $\mathrm{H}$, and $\mathrm{Li}$, J, Intranasal Dexmedetomidine on Stress Hormones, Inflammatory Markers, and Postoperative Analgesia after Functional Endoscopic Sinus Surgery. Mediators Inflamm, 2015. 2015: p. 939431.

19. Tasdogan M, Memis D, Sut N, Yuksel M. Results of a pilot study on the effects of propofol and dexmedetomidine on inflammatory responses and intraabdominal pressure in severe sepsis. J Clin Anesth. 2009; 21:394-400.

20. Xianbao, L.; Hong, Z.; Xu, Z.; Chunfang, Z.; Dunjin, C. Dexmedetomidine reduced cytokine release during postpartum bleedinginduced multiple organ dysfunction syndrome in rats. Mediat. Inflamm. 2013, 2013, 627831.

21. Yacout, A., Osman, H, Abdeldaem, M, Hammouda, S. Effect of intravenous dexmedetomidine infusion on some proinflammatory cytokines, stress hormones and recovery profile in major abdominal surgery. Alexandria Journal of Medicine 2012, 
SOHAG MEDICAL JOURNAL

Vol. 21 No.2 July 2017
Hemodynamic and analgesic effects Islam Mokhtar Ahmed.et al 